\title{
Design for realizing arbitrary fractional divider based on FPGA which duty cycle is up to $50 \%$
}

\author{
ZHANG Song-wei \\ Dept. of Electronics and Communications Engineering, \\ Zhengzhou Institute of Aeronautical Industry Management, \\ Zhengzhou 450015, China. \\ e-mail: netsurfersw@163.com
}

\author{
ZHAO Cheng \\ Dept. of Electronics and Communications Engineering, \\ Zhengzhou Institute of Aeronautical Industry Management, \\ Zhengzhou 450015, China. \\ e-mail: ziapc@yahoo.cn
}

\begin{abstract}
This paper proposes a novel method for realizing arbitrary fractional divider based on FPGA. Analyzing the limitations of the existing frequency-divided methods, a new model which consists of two-level dividers is put forward. An arbitrary frequency-divided clock output can be obtained by this method approaching $50 \%$ of duty cycle. When the division factor is greater than 128 , the duty cycle can be very close to $50 \%$ of the clock output. This method is proved to be feasible on the FPGA chip of ALTERA.
\end{abstract}

Keywords- fractional divider; FPGA; duty cycle

\section{INTRODUCTION}

In modern communication systems, clock is more and more important as a vital frequency resource. In the system, clocks of different levels are needed in response to equipments of different classes, which need to be correspondingly divided aiming at the system clocks. According to the needs of different designs, we will encounter integer, half-integer, arbitrary fractional divider, etc [Ref.1-5]. Several forms of frequency division appear even in the same design. Generally speaking, the duty cycle of clocks is up to $50 \%$. It is easy to implement when frequency divider ratio is even, while in other cases, it is more difficult $[6,8]$. This article, inspired by the design philosophy of the universal half-integer frequency divider, proposes a general design method and achieves the goal of arbitrary frequency of equal duty ratio. With the use of hardware description language of Verilog HDL, development platform of Quartus II 9.0 and Altera's FPGA, this article designs a more common fractional divider to fulfill all the above requirements.

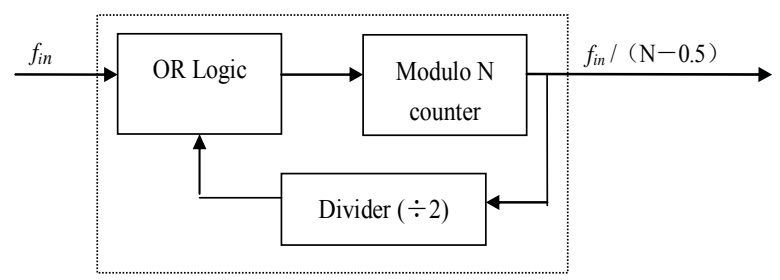

Figure 1. Half-integral frequency divider

Half-integer divider usually consists of a modulo $\mathrm{N}$ counter, a divide-by-2 divider and an OR logic, as shown in Figure 1.
The principle can be implemented on the basis of $\mathrm{N}$ counters, by means of deducting half cycle of nonfrequency clock by Exclusive OR, so as to achieve the N0.5 count, $\mathrm{N}-0.5$ frequency division and realize that the circuit of deduction is made up of a divide-by-2 divider and an Exclusive OR. Odd frequency $(2 N-1)$ can be achieved and the duty cycle is up to $50 \%$ if the output of the divide-by-2 divider can output signal. It is visible that $50 \%$ duty cycle is because of divide-by-2, which can be implemented by a trigger. Based on this, we can construct an arbitrary divider of frequency factor for $K$ (as shown in Figure 2), and reach or try to approximate $50 \%$ duty cycle.

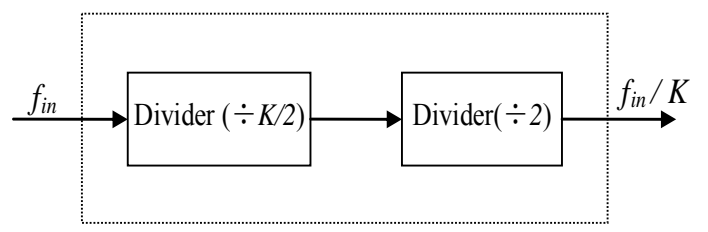

Figure 2. arbitrary divider of frequency factor for $\mathrm{K}$

When $K$ takes different numeric values, the $K / 2$ divider will appear the situations of an integer divider, half-integer divider and fractional divider. The following are discussed respectively.

\section{INTEGER DIVIDER AND HALF-INTEGER DIVIDER}

\section{A. Integer divider}

When $K$ equals to $2 N$ in which $\mathrm{N}$ is an integer, that is, when $K$ is even, $K / 2$ divider changes into an integer divider (as shown in Figure 3). Two types of methods can be achieved for the FPGA design of an integer frequency divider. One is to achieve by using a counter, the other is to implement with the use of state machines. The two methods have their own merits. Counter is simple to use and occupies less resource. There may be a number of changes of he outputs of the normal counter, so while using combinational logic to decode the output, because of the issues of signal competition and adventure, the normal counter will cause the peak pulse. Using Johnson counter can avoid the problem, as only one output of it changes under each clock. Sate machines have the advantage of clear levels of description, but occupy more resources. So it can be carefully used in specific designs. 
For an even divider, it can be up to $50 \%$ duty cycle, as shown in Figure 3.

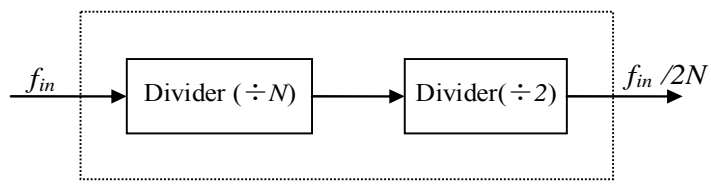

Figure 3. Even divider $(K=2 N)$

\section{B. Half-integer divider}

When $K$ equals to $2 N-1$ in which $N$ is an integer, that is, when $\mathrm{k}$ is odd, $K / 2$ divider changes into a half-integer divider $(N-0.5)$.

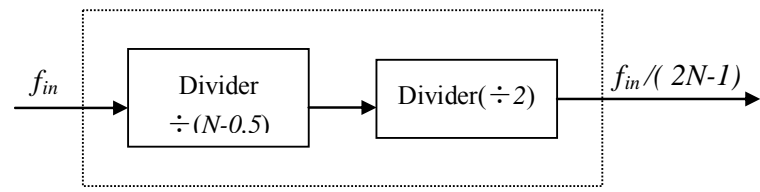

Figure 4. Fig 4 Odd divider $(\mathrm{K}=2 \mathrm{~N}-1)$

Actually we can choose the method as shown in Figure 1 , odd frequency signal of $50 \%$ duty cycle can be obtained if the output of the divide-by-2 divider can directly output signal. Simulation waveform is shown in Figure 5 in which the wave of outclk1 and outclk2 can be seen in the figure that outclk1 signals producing a rising edge every 3.5 clock cycles achieves frequency of coefficient for 3.5, at the same time, gets a 7 frequency of duty cycle on the outclk2 side.

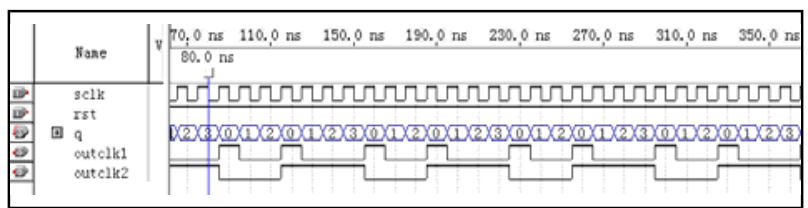

Figure 5. Odd frequency timing simulation diagram $(K=2 N-1, N=$ 4).

\section{FRACTIONAL DIVIDER}

When the frequency coefficient $K$ is an arbitrary fraction, $K / 2$ divider is a fractional divider. If $K$-divided frequency is directly divided, the duty cycle of the output signal varies widely, or deviates more than $50 \%$ and is uneven. It can be finally reached $K$-divided frequency of approaching $50 \%$ duty cycle by applying first divided-by$K / 2$ frequency and again divided-by-2 frequency.

\section{A. Principles of fractional divider}

So-called fractional divider is only an averaged effect; the coefficient of every frequency is an integer. Under the effect of the control logic, it is merely switched between the two frequencies. The same as the basic principle of realizing fractional divider, a certain way in several frequency cycles is taken to make a few cycles count more than or less than a number, so as to obtain a fractional divider ratio in overall count cycle of entirely average significance, as shown in Figure 6. Suppose a fractional divider of frequency coefficient for $\mathrm{K}$ is to be divided, $\mathrm{K}$ can be expressed as:

$$
K=\frac{f_{\text {in }}}{f_{\text {out }}}=N+\frac{n_{2}}{n_{1}+n_{2}}=\frac{n_{1} N+n_{2}(N+1)}{n_{1}+n_{2}} .
$$

In the formula, $n_{1}, n_{2}, N$, are all positive integers. Formula (1) has a clearly physical meaning and implementation methods of engineering. In $n_{1}+n_{2}$ times of dividing frequency, $N$-divided frequency divides $n_{1}$ times, $(N+1)$-divided frequency $n_{2}$ times, and the average effects of $n_{1}+n_{2}$ times of dividing frequency can be achieved to the effects of fractional divider. For example, if you want to get a frequency coefficient of $\mathrm{K}$ for 3.7, you just apply divided-by-4 frequency of 7 times and dividedby-3 frequency of 3 times, so the average frequency ratio $=(3 \times 3+4 \times 7) /(3+7)=3.7$. As another example, $K$ is frequency coefficient for $3.18181818 \cdots \cdots$, that is, when $K=3+\frac{2}{11}$, it is enough to divide by 4 frequency of 2 times and divide by 3 frequency of 9 times. The times of divided-by- $N$ and divided-by- $(N+1)$ frequency have nothing to do with the integer portion. For a fractional divider exists two frequencies of divided-by- $\mathrm{N}$ and divided-by- $(N+1)$, and each of which divides many times, the two frequencies should be attempted to be mixed well, rather than all divided-by- $N$ frequency over a period of time, divided-by- $(N+1)$ frequency at another time, otherwise, large phase fluctuations will happen. There are two mixed methods:

- $\quad$ The first method:

Step 1: transforming the fractional part of the fractional divider into type of fractional: $\frac{n_{2}}{n_{1}+n_{2}}$.

Step 2: Each frequency cumulates $n_{2}$. If it is less than $n_{1}+n_{2}$, then divides by $N$ frequency, meanwhile, do some adjustments to the accumulated value, minus $n_{1}+n_{2}$, or else divide by $(N+1)$ frequency.

- The second method:

Step 1: the same with step1 of the first method.

Step 2: The accumulated value is set up to $n_{1}$, if is less than $n_{1}+n_{2}$ after accumulation, then divides by $(N+1)$ frequency, otherwise, divide by $N$ frequency. Meanwhile, do some adjustments to the accumulated value, minus $n_{1}+n_{2}$. 
[6] MAO Weiyong, QI Zhongyang,WANG Lan. Design for decimal frequency divider based on FPGA[J]. Joural of GuiLin College of Aerospace Technology, vol.53 (1), Jan. 2009, pp.30 -32.

[7] ZHANG Jianyu, Sun Chengshou, LAI Jinmei. "Design and Realization of synthesizer programmable divider for $2.4 \mathrm{GHz}$ frequency," Journal of Fudan University: Natural Science Edition, vol. 44 (1), Jan. 2005, pp.139.

[8] ZHOU Dongcheng, WANG Yongbin, ZHENG Yaping. "Design of Fractional-N Frequency Synthesizer based on FPGA," Electronic Measurement Technology, vol.29(3), Mar. 2006, pp.79-93. 